\title{
LOCAL MONODROMY OF BRANCHED COVERS AND DIMENSION OF THE BRANCH SET
}

\author{
Martina Aaltonen and Pekka Pankka \\ University of Helsinki, Department of Mathematics and Statistics \\ P. O. Box 68, FI-00014 University of Helsinki, Finland; martina.aaltonen@helsinki.fi \\ University of Helsinki, Department of Mathematics and Statistics \\ P. O. Box 68, FI-00014 University of Helsinki, Finland; pekka.pankka@helsinki.fi
}

\begin{abstract}
We show that, if the local dimension of the image of the branch set of a discrete and open mapping $f: M \rightarrow N$ between $n$-manifolds is less than $(n-2)$ at a point $y$ of the image of the branch set $f B_{f}$, then the local monodromy of $f$ at $y$ is perfect. In particular, for generalized branched covers between $n$-manifolds the dimension of $f B_{f}$ is exactly $(n-2)$ at the points of abelian local monodromy. As an application, we show that a generalized branched covering $f: M \rightarrow N$ of local multiplicity at most three between $n$-manifolds is either a covering or $f B_{f}$ has local dimension $(n-2)$.
\end{abstract}

\section{Introduction}

A continuous mapping $f: X \rightarrow Y$ between topological spaces is a (generalized) branched cover if $f$ is discrete and open, that is, pre-image $f^{-1}(y)$ of a point $y \in Y$ is a discrete set and $f$ maps open sets to open sets. The name branched cover for these maps stems from the Chernavskii-Väisälä theorem [5, 18]: the branch set of a branched cover between (generalized) manifolds has codimension at least two. It is an easy consequence of the Chernavskii-Väisälä theorem that branched covers between (generalized) manifolds are, at least locally, completions of covering maps.

We follow here the typical naming convention in this context and say that a point $x \in X$ is a branch point of $f$ if $f$ is not a local homeomorphism at $x$. The branch set of the mapping $f$, i.e. the set of branch points of $f$, is denoted $B_{f}$. Note that, in the context of PL topology, $B_{f}$ is called the singular set and its image $f B_{f}$ the branch set.

Branch sets of branched covers between surfaces are well-understood. By the classical Stoïlow theorem (see e.g. [19]), the branch set of a branched cover between surfaces is a discrete set. In higher dimensions, branch sets of PL branched covers between manifolds are subcomplexes of codimension at least two. More general branched covers may, however, exhibit also wilder branching behavior. Heinonen and Rickman constructed in [12] and [13] quasiregular, even BLD, branched covers $S^{3} \rightarrow S^{3}$ which contain wild Cantor sets in their branch sets; see also [17]. In fact, in dimensions $n \geq 3$, branch sets of branched covers are not understood in a similar precise fashion as in two dimensions. In particular a conjecture of Church and Hemmingsen [7] is still open: The branch set of a branched cover between 3-manifolds has topological dimension one.

https://doi.org/10.5186/aasfm.2017.4231

2010 Mathematics Subject Classification: Primary 57M12; Secondary 57M30, 30C65.

Key words: Branched cover, monodromy, branch set.

M. A. has been supported by the Emil Aaltonen foundation and P. P. partly supported by the Academy of Finland project 256228. 
It is easy to observe that the conjecture of Church and Hemmingsen is equivalent to the question whether there exists a branched cover between 3-manifolds for which $f B_{f}$ is a wild Cantor set in a neighborhood of a point in $f B_{f}$; see also Church [6] and Montesinos [16] for related questions. Note that, we have $\operatorname{dim} B_{f}=\operatorname{dim} f^{-1} B_{f}=$ $\operatorname{dim} f B_{f}$ for branched covers $f: M \rightarrow N$ between manifolds by [7, Corollary 2.3].

In this article we consider the connection of the monodromy to the local dimension of the branch set. This question is interesting already in the context of PL branched covers as the following example shows.

Let $f: S^{3} \rightarrow H^{3}$ be a normal covering of the Poincaré homology sphere $H^{3}$ and $F=\Sigma^{2} f: \Sigma^{2} S^{3} \rightarrow \Sigma^{2} H^{3}$ the double suspension of $f$. Then $F$ is a normal branched cover for which $B_{F}$ is a circle in $S^{5}=\Sigma^{2} S^{3}$ and $F B_{F}$ in $\Sigma^{2} H^{3} \cong S^{5}$ is a wild knot; see Edwards [9] and Cannon [4]. In particular, $B_{F}$ and $F B_{F}$ both have codimension 4. The monodromy group $G_{F}$ of $F$ is isomorphic to the fundamental group of $H^{3}$ which is a perfect group. Recall, that a group $\Gamma$ is perfect if $\Gamma /[\Gamma, \Gamma]$ is a trivial group. Our main result shows that this is a general phenomenon: If the branch set of a branched cover has codimension larger than two, then the local monodromy groups of the map are perfect.

We define the local monodromy of a branched cover as follows. Let $f: M \rightarrow N$ be a proper branched cover between $n$-manifolds. By a result of Berstein and Edmonds [2] (see also [1]), there exists a space $X_{f}$ and an action $G_{f} \curvearrowright X_{f}$ of the monodromy group $G_{f}$ of $f$ by homeomorphisms for which the diagram

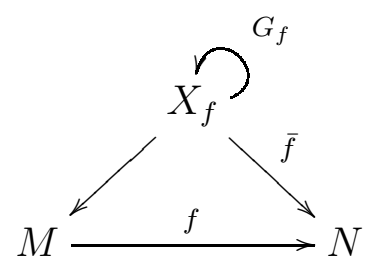

commutes, where the maps $X_{f} \rightarrow M$ and $\bar{f}: X_{f} \rightarrow N$ are normal branched covers. We call $X_{f}$ the monodromy space of $f$. Recall that, similarly as for covering maps, a branched cover $h: X \rightarrow Y$ is normal if $h$ is a quotient map of the action of the deck group $\operatorname{Deck}(h)$ to $X$.

We call the map $\bar{f}: X_{f} \rightarrow N$, which is the orbit map of the action $G_{f} \curvearrowright X_{f}$, the normalization of $f$; in particular, $\operatorname{Deck}(\bar{f})=G_{f}$. Given $y \in N$, the stabilizer subgroups of $G_{f}$ of points in $\bar{f}^{-1}(y)$ are conjugate to each other and we define the local monodromy $\mathcal{G}_{f}(y)$ of $f$ at $y \in N$ to be the conjugacy class of these subgroups.

Our main theorem reads as follows.

Theorem 1. Let $f: M \rightarrow N$ be a proper branched cover between $n$-manifolds. If the local dimension of $f B_{f}$ at $y \in f B_{f}$ is less than $n-2$, then $\mathcal{G}_{f}(y)$ is a finite perfect group.

As an immediate corollary of Theorem 1 and the Chernavskii-Väisälä theorem on the dimension of the branch set, we obtain an elementary proof for the following well-known result; for a proof using the Smith theory, see e.g. [3].

Corollary 2. Let $f: M \rightarrow N$ be a proper normal branched cover between $n$ manifolds having abelian deck group. Then either $f$ is a covering map or $\operatorname{dim} B_{f}=$ $\operatorname{dim} f B_{f}=n-2$. 
As an application of Theorem 1, we also obtain a positive result in a special case of the conjecture of Church and Hemmingsen for branched covers having local multiplicity at most three. More precisely we have the following result.

Theorem 3. Let $f: M \rightarrow N$ be a proper branched cover between $n$-manifolds so that the local multiplicity of $f$ is at most three in $B_{f}$. Then either $f$ is a covering map or $f B_{f}$ has local dimension $n-2$.

A remark on the relation of these results to the classical Smith theory is in order. For normal branched covers $f: M \rightarrow N$ between (cohomology) manifolds, the branch set $B_{f}$ has decomposition into finitely many cohomology manifolds of codimension at least 2 (see e.g. [3, Theorem V.2.2]). Since $B_{f}$ is not a (cohomology) 0-manifold, it is therefore easy to conclude that $f B_{f}$ has local dimension at least 1 at each point. It is not known to us to which extent these methods are available in the context of Theorem 1, since the monodromy space $X_{f}$ of $f$ is not a priori a cohomology $n$-manifold.

We finish this introduction with a non-existence result for branched covers branching over an Antoine's necklace. A branched cover $f: X \rightarrow Y$ is locally normal if each point $x \in X$ has a neighborhood $U$ for which $\left.f\right|_{U}: U \rightarrow f U$ is a normal branched cover.

Theorem 4. There are no locally normal branched covers $S^{3} \rightarrow S^{3}$ for which the image of the branch set is an Antoine's necklace.

This article is organized as follows. After discussing preliminaries in Section 2, we prove in Section 3 a slightly more general version of Theorem 1 for branched covers from Fox-completions of manifolds to manifolds. In Section 4 we give a proof of a similar generalization for Theorem 3. Finally, in Section 5, we discuss applications to Cantor sets and prove Theorem 4.

Acknowledgments. Authors thank Juan Souto and Jang-Mei Wu for discussions and their comments on the manuscript.

\section{Preliminaries}

In this section, we recall a few basic facts on branched covers and introduce some terminology which we use in the forthcoming sections. Note that we consider only path-connected manifolds and manifold completions.

2.1. Coverings. Let $f: X \rightarrow Y$ be a covering map between path-connected spaces and $y_{0} \in Y$. The monodromy $\mu_{f}: \pi_{1}\left(Y, y_{0}\right) \rightarrow \operatorname{Sym} f^{-1}\left(y_{0}\right)$ of $f$ is the homomorphism which associates a permutation of $\operatorname{Sym} f^{-1}\left(y_{0}\right)$ to every homotopy class $[\gamma]$. More precisely, let $\gamma:[0,1] \rightarrow Y$ be a loop based at $y_{0}, x \in f^{-1}\left(y_{0}\right)$, and $\tilde{\gamma}_{x}:[0,1] \rightarrow X$ the lift of $\gamma$ from $x$ in $f$. Then $\mu_{f}([\gamma])(x)=\tilde{\gamma}_{x}(1)$. The monodromy group $G_{f}$ of $f$ is the quotient $\pi_{1}\left(Y, y_{0}\right) / \operatorname{ker} \mu_{f}$.

For normal coverings $f:\left(X, x_{0}\right) \rightarrow\left(Y, y_{0}\right)$ of pointed spaces, we have also the deck homomorphism $\sigma_{f, x_{0}}: \pi_{1}\left(Y, y_{0}\right) \rightarrow \operatorname{Deck}(f)$ of $f$ which associates to each homotopy class $[\gamma] \in \pi_{1}\left(Y, y_{0}\right)$ a deck transformation using the lift of the representative $\gamma$ from $x_{0}$, that is, given a loop $\gamma:[0,1] \rightarrow Y$ at $y_{0}$, we set $\sigma_{f, x_{0}}([\gamma])$ to be the (unique) deck transformation $\tau: X \rightarrow X$ satisfying $\tau(x)=\tilde{\gamma}_{x_{0}}(1)$. Note that, $G_{f} \cong \operatorname{Deck}(f)$, but typically, the deck transformation $\sigma_{f, x_{0}}([\gamma])$ is not an extension of the permutation $\mu_{f}([\gamma])$. 
2.2. Manifold completions and proper branched coverings. We say that a locally connected (and locally compact) Hausdorff space $X$ is a Fox-completion of a manifold, if there exists a connected $n$-manifold $X^{o}$ (possibly with boundary) and an embedding $\iota: X^{o} \hookrightarrow X$, so that $\iota\left(X^{o}\right) \subset X$ is dense and the set $X \backslash \iota\left(X^{o}\right)$ does not locally separate $X$. In particular, $\overline{\iota\left(X^{o}\right)}=X$. We refer to Fox [11] and Montesinos [16] for further details.

This class of spaces rises naturally in the context of branched covers. Indeed, if $f: X \rightarrow M$ is a branched cover from a locally compact and locally connected Hausdorff space $X$ to an $n$-manifold $M$ so that $B_{f}$ does not locally separate $X$. Then $X$ is a Fox-completion of a manifold, since $X^{o}=X \backslash B_{f}$ is an $n$-manifold.

Let $f: X \rightarrow M$ be a proper branched cover from a Fox-completion $X$ of a manifold to a manifold $M$. Then $f$ is a completed cover, that is, $f$ is the unique extension of the covering $f^{\prime}=\left.f\right|_{X^{\prime}}: X^{\prime} \rightarrow M^{\prime}$ with respect to $M^{\prime}$, where $X^{\prime}=$ $X \backslash f^{-1} f B_{f}$ and $M^{\prime}=M \backslash f B_{f}$ are open dense subsets of $X$ and $M$, respectively, and the sets $f^{-1} f B_{f}$ and $f B_{f}$ do not locally separate $X$ and $M$, respectively. Indeed, since $f$ is proper, $f$ is surjective and both $f B_{f}$ and $f^{-1} f B_{f}$ are closed sets. For the general theory of these completions, see e.g. Fox [11], Berstein-Edmonds [2], Edmonds [8], or [1]. We call $f^{\prime}$ the regular part of $f$.

We recall two facts on proper branched covers. First, $f: X \rightarrow M$ is a proper normal branched cover if and only if its regular part $f^{\prime}: X^{\prime} \rightarrow M^{\prime}$ is a proper normal covering; see Edmonds [8]. We also recall that the homomorphism $\operatorname{Deck}(f) \rightarrow \operatorname{Deck}\left(f^{\prime}\right)$, $\left.g \mapsto g\right|_{X^{\prime}}$, is an isomorphism; see Montesinos [15].

We define the monodromy of $f: X \rightarrow M$ to be the monodromy of its regular part $f^{\prime}: X^{\prime} \rightarrow M^{\prime}$, that is,

$$
\mu_{f}:=\mu_{f^{\prime}}: \pi_{1}\left(M^{\prime}, y_{0}\right) \rightarrow \operatorname{Sym} f^{-1}\left(y_{0}\right)
$$

for $y_{0} \in M^{\prime}$.

2.3. Monodromy triangle. The regular part $f^{\prime}: X^{\prime} \rightarrow M^{\prime}$ of a branched covering $f: X \rightarrow M$ has, by the classical covering space theory, a monodromy triangle

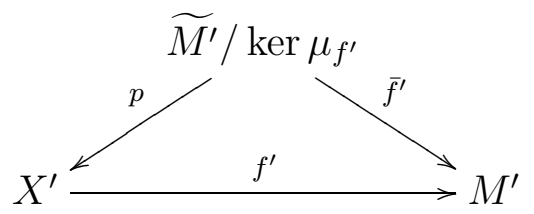

where the normal covering map $\bar{f}^{\prime}$ is the orbit map of the natural action of the monodromy group $G_{f^{\prime}}=\pi_{1}\left(M^{\prime}, y_{0}\right) / \operatorname{ker} \mu_{f^{\prime}}$ on $\widetilde{M}^{\prime} /$ ker $\mu_{f^{\prime}}$ by homeomorphisms. The monodromy triangle (1) of $f: X \rightarrow M$ is obtained as an extension of the monodromy triangle of its regular part $f^{\prime}: X^{\prime} \rightarrow M^{\prime}$. We refer to Berstein-Edmonds [2], or [1], for details.

Note that, given a normalization $\bar{f}: X_{f} \rightarrow M$ of a proper branched cover $f: X \rightarrow$ $M$ and a subgroup $H \subset G_{f}$ of the monodromy group $G_{f}$ of $f$, there exists a factorization

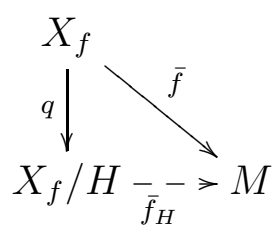

where $q$ and $\bar{f}_{H}$ are branched covers induced by the action $H \curvearrowright X_{f}$. Moreover, if $H$ is normal in $G_{f}$, then $\bar{f}_{H}$ is a normal branched cover with $\operatorname{Deck}\left(\bar{f}_{H}\right) \cong \operatorname{Deck}(\bar{f}) / H$. 
2.4. Regular neighborhoods. For the localization arguments we recall the existence of normal neighborhoods (Väisälä [18, Lemma 5.1]): Let $f: X \rightarrow M$ be a branched cover from a Fox-completion $X$ of a manifold to a manifold $M$. Then, for each $x \in X$, there exists a neighborhood $U \subset X$ of $x$ for which $\left.f\right|_{U}: U \rightarrow f U$ is a proper branched cover. Moreover, for each domain $V$ compactly contained in $f U$ and each component $W \subset f^{-1} V \cap U$, the restriction $\left.f\right|_{W}: W \rightarrow V$ is a proper branched cover.

2.5. Topological dimension and cohomology. In what follows, we call the covering dimension of a space simply as dimension. Recall that a space $X$ has covering dimension at most $n$ (denoted $\operatorname{dim} X \leq n$ ) if each covering of $X$ has a refinement of local multiplicity at most $n+1$. Further, $X$ has dimension $n(\operatorname{dim} X=n)$ if $\operatorname{dim} X \leq n$ and $X$ does not have covering dimension at most $n-1$. See e.g. Engelking [10] for comparisons with other definitions.

A closed set $E \subset X$ has local dimension at most $n$ at $x \in E$ if there exists a neighborhood $U \subset X$ of $x$ so that $\operatorname{dim}(U \cap E) \leq n$. Similarly, $E$ has local dimension at least $n$ if for all neighborhoods $U \subset X$ of $x$ for which $\operatorname{dim}(U \cap E) \geq n$.

In the proof of Theorem 6 we use the fact that the Alexander-Spanier (or equivalently Cech-cohomology) groups $H^{k}(X ; \mathbf{Z})$ are trivial for $k>n$ if $\operatorname{dim} X \leq n$; see e.g. [10, pp. 94-95]. Note that a Fox-completion of a manifold is a Cantor manifold in the sense of [10, Definition 1.9.5].

\section{Proof of Theorem 1}

In this section we prove the following version of Theorem 1 .

Theorem 5. Let $X$ be a Fox-completetion of an $n$-manifold, $M$ an $n$-manifold, $f: X \rightarrow M$ a proper branched cover and $y \in f B_{f}$. If the local dimension of $f B_{f}$ at $y \in f B_{f}$ is less than $n-2$, then $\mathcal{G}_{f}(y)$ is a finite perfect group.

We begin with the abelian case of the theorem.

Theorem 6. Let $X$ be a Fox-completion of an $n$-manifold, $M$ an $n$-manifold, and $f: X \rightarrow M$ a proper branched cover for which $B_{f} \neq \emptyset$. The local dimension of $f B_{f}$ is $(n-2)$ at points of abelian local monodromy.

By Chernavskii-Väisälä theorem, the local dimension of $f B_{f}$ is at most $n-2$. Thus we may assume in this section that $n \geq 3$ and show that the dimension of $f B_{f}$ is at least $n-2$ at points of abelian local monodromy.

Remark 7. There are simple examples of branched covers having points where the local monodromy is abelian and not cyclic. For example, let $f: \mathbf{R}^{3} \rightarrow \mathbf{R}^{3}$ be the composition $f=f_{1} \circ f_{2}$, where $f_{1}: \mathbf{R}^{3} \rightarrow \mathbf{R}^{3}$ is a 2-to- 1 winding map around the $x$-axis and $f_{2}: \mathbf{R}^{3} \rightarrow \mathbf{R}^{3}$ is a 2 -to- 1 winding map around the $y$-axis. Then $f$ is a proper 4-to-1 normal branched cover for which the local monodromy at the origin is the abelian, but non-cyclic group, $\mathbf{Z}_{2} \times \mathbf{Z}_{2}$. At every other point of $f B_{f}$ the local monodromy group is the cyclic group $\mathbf{Z}_{2}$.

Proof of Theorem 6. Suppose there exists $y \in f B_{f}$ for which the local dimension of $f B_{f}$ at $y$ is less than $n-2$. Let $\bar{f}: X_{f} \rightarrow M$ be a normalization of $f$ and $x \in \bar{f}^{-1}(y)$. We show first that there exists a neighborhood $U \subset X$ of $x$, having closure $E=\bar{U}$, so that $\bar{f} E$ is an $n$-cell in $M$, and $\left.\bar{f}\right|_{E}: E \rightarrow \bar{f} E$ is a proper branched cover and $\operatorname{dim}\left(\left.\bar{f}\right|_{E} B_{\left.\bar{f}\right|_{E}}\right)<n-2$. 
Let $W$ be a neighborhood of $y$ in $M$ contained in an $n$-cell and for which $\operatorname{dim}(W \cap$ $\left.f B_{f}\right)<n-2$. Then, by [18, Lemma 5.1], we may fix a neighborhood $V \subset X$ of $x$ so that $f V \subset W$ and $\left.f\right|_{V}: V \rightarrow f V$ is a proper map. Let $D \subset \operatorname{int}(f V \backslash f(\partial V))$ be an open $n$-cell so that $\bar{D}$ is an $n$-cell. Then $\left.f\right|_{\bar{U}}: \bar{U} \rightarrow \bar{D}$, where $U$ is the $x$-component of $f^{-1} D$, is a proper branched cover.

We construct now a double of $\left.\bar{f}\right|_{E}: E \rightarrow \bar{f} E$ as follows. Let $Z$ be the quotient space obtained by gluing two copies of $E$ together along the boundary. More precisely, let $Z=(E \times\{1,2\}) / \sim$, where $\sim$ is the minimal equivalence relation satisfying $(x, 1) \sim(x, 2)$ for $x \in \partial E$. Let $q_{E}: E \times\{1,2\} \rightarrow Z$ be the quotient map $(x, i) \mapsto$ $[(x, i)]$. The non-manifold points of $Z$ are contained in the set $q_{E}\left(\left(B_{\bar{f}} \cap E\right) \times\{1,2\}\right)$ and $Z$ is a Fox-completion of an $n$-manifold.

Let also $S=((\bar{f} E \times\{1\}) \cup(\bar{f} E \times\{2\})) / \sim$ be an $n$-sphere obtained by gluing the $n$-cells $\bar{f} E \times\{i\}$ together along the boundary $\partial \bar{f} E$ similarly as above. Let $q: \bar{f} E \times\{1,2\} \rightarrow S$ be the associated quotient map.

Let $g: Z \rightarrow S$ be the unique map for which the diagram

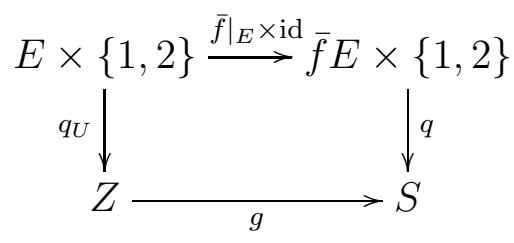

commutes. Then $g$ is an open and discrete map. Indeed, discreteness of $g$ follows immediately. For the openness of $g$, it suffices to observe that, given a neighborhood $V$ of a point in $q_{E}(\partial E \times\{1\})$, there exists open sets $V_{1}$ and $V_{2}$ in $E$ so that $q_{E}\left(\left(V_{1} \times\right.\right.$ $\left.\{1\}) \cup\left(V_{2} \times\{2\}\right)\right)=V$. Then

$$
g V=q\left(\left(\bar{f} V_{1} \times\{1\}\right) \cup\left(\bar{f} V_{2} \times\{2\}\right)\right)
$$

is an open set in $S$. Thus $g$ is a branched cover. Similarly we observe that $g$ is, in fact, a normal branched cover having an abelian deck group $\operatorname{Deck}(g) \cong \operatorname{Deck}\left(\left.\bar{f}\right|_{E}\right)$ and $g B_{g} \subset q\left(\left.\bar{f}\right|_{E} B_{\left.\bar{f}\right|_{E}} \times\{1,2\}\right)$.

Since $\operatorname{dim}\left(g B_{g}\right)<n-2$ and $n \geq 3$, we have, by the Alexander duality (see e.g. [14, Theorem 6.6]), that

$$
H_{1}\left(S^{n} \backslash g B_{g}\right) \cong H^{n-2}\left(g B_{g} ; \mathbf{Z}\right)=0 .
$$

Thus $\pi_{1}\left(S^{n} \backslash g B_{g}, z_{0}\right)$ is a perfect group for every $z_{0} \in S^{n} \backslash g B_{g}$.

Let

$$
g^{\prime}:=g \mid Z \backslash g^{-1}\left(g B_{g}\right): Z \backslash g^{-1}\left(g B_{g}\right) \rightarrow S^{n} \backslash g B_{g}
$$

be a restriction of $g$ and let $\varphi_{g^{\prime}, y_{0}}: \pi_{1}\left(S^{n} \backslash g B_{g}, z_{0}\right) \rightarrow \operatorname{Deck}\left(g^{\prime}\right)$ be the deck-homomorphism for points $z_{0} \in S^{n} \backslash g B_{g}$ and $y_{0} \in g^{\prime-1}\left\{z_{0}\right\}$. Then $\operatorname{Deck}\left(g^{\prime}\right) \cong \operatorname{Deck}(g)$ is abelian. Thus Deck $\left(g^{\prime}\right)$ is an abelian image of a perfect group, and hence trivial. We conclude that then $\operatorname{Deck}(g)$ is also trivial and the normal branched cover $g$ is a homeomorphism. Hence also $\left.\bar{f}\right|_{E}$ is a homeomorphism and $\mathcal{G}_{f}(y)$ is trivial. This is a contradiction, since $y \in f B_{f} \cap U$. Hence the local dimension of $f B_{f}$ at each of its points is at least $n-2$. 
Proof of Theorem 5. Suppose the local dimension of $f B_{f}$ at $y \in f B_{f}$ is less than $n-2$. Let $G_{f}$ be the monodromy group of $f$ and let

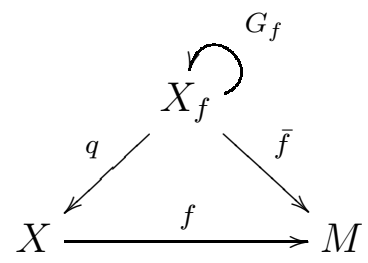

be the monodromy triangle of $f$, where $\bar{f}$ is the normalization of $f$. We need to show that $\mathcal{G}_{\bar{f}}(z)=\left[\mathcal{G}_{\bar{f}}(z), \mathcal{G}_{\bar{f}}(z)\right]$ for a point $z \in \bar{f}^{-1}(y)$.

Let $V_{0}$ be such a neighborhood of $y$ that the dimension of $V_{0} \cap f B_{f}$ is less than $n-2$ and let $z \in \bar{f}^{-1}(y)$. By Väisälä's lemma [18, Lemma 5.1], we may fix a neighborhood $W$ of $z$ for which $\bar{f} W \subset V_{0}$ is simply connected, $\bar{f}^{-1}(\bar{f}(z))=\{z\}$, and that the restriction $\left.\bar{f}\right|_{W}: W \rightarrow \bar{f} W$ is a proper branched cover. We denote $U=q W$ and $V=\bar{f} W$. Then $g:=\left.f\right|_{U}: U \rightarrow V$ is a proper branched cover and we have the diagram

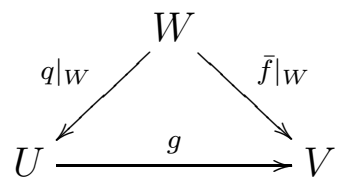

where $q \mid W$ and $\bar{f} \mid W$ are normal branched coverings and $\mathcal{G}_{\bar{f}}(z) \cong \operatorname{Deck}\left(\left.\bar{f}\right|_{W}\right)$.

Let $h=\left.\bar{f}\right|_{W}: W \rightarrow V$, and denote $N=[\operatorname{Deck}(h)$, $\operatorname{Deck}(h)]$. We factor the normal branched cover $h$ as

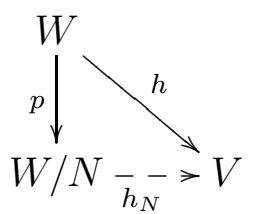

where $p: W \rightarrow W / N$ is the quotient map $x \mapsto N x$. Since $N \subset \operatorname{Deck}(h)$ is normal, $h_{N}: W / N \rightarrow V$ is a normal branched covering and $\operatorname{Deck}\left(h_{N}\right) \cong \operatorname{Deck}(h) / N$. Hence $\operatorname{Deck}\left(h_{N}\right)$ is abelian. Since $\left(h_{N}\right) B_{h_{N}} \subset f B_{f}$ and the dimension of $V \cap f B_{f}$ is less than $n-2$, we have by Theorem 6 that $h_{N}$ is a covering map. Since $V$ is simply-connected, $h_{N}$ is a homeomorphism. Thus $\operatorname{Deck}\left(\left.\bar{f}\right|_{W}\right)=N$.

\section{Proof of Theorem 3}

We prove now a version of Theorem 3 for branched covers having a Fox-completion of a manifold as a domain. The statement reads as follows.

Theorem 8. Let $f: X \rightarrow M$ be a proper branched cover from a Fox-completion $X$ of an $n$-manifold to an $n$-manifold $M$ so that the local multiplicity of $f$ is at most 3 in $B_{f}$. Then either $f$ is a covering or $f B_{f}$ has local dimension $n-2$.

Proof. Suppose that $f$ is not a covering map. We show that the local dimension of $f B_{f}$ is $n-2$ at each point of $f B_{f}$. By the Chernavskii-Väisälä theorem, the local dimension of $f B_{f}$ is at most $n-2$ at each point $y \in f B_{f}$. Thus it suffices to show that the local dimension of $f B_{f}$ at $f(x)$ is at least $n-2$.

Suppose first that there exists $x \in B_{f}$ for which the local multiplicity of $f$ at $x$ is 2. Then $\mathcal{G}_{f}(f(x)) \cong \mathbf{Z}_{2}$ and, by Theorem 6 , the local dimension of $f B_{f}$ is $n-2$. 
Suppose now that the local multiplicity of $f$ at $x \in B_{f}$ is 3 . Let $G_{f}$ be the monodromy group of $f$ and let

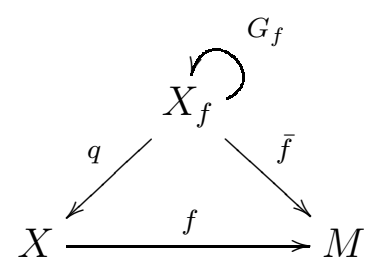

be the monodromy triangle of $f$, where $\bar{f}$ is the normalization of $f$.

Let $U_{0}$ be a neighborhood of $x$ so that $\left.f\right|_{U_{0}}: U_{0} \rightarrow M$ has multiplicity at most 3 . Let now $z \in q^{-1}(x)$. As in the proof of Theorem 1 we fix, using Väisälä's lemma [18, Lemma 5.1], a neighborhood $W$ of $z$ so that $\left.\bar{f}\right|_{W}: W \rightarrow \bar{f} W$ is a proper branched cover, $\bar{f} \mid W^{-1}(\bar{f}(z))=\{z\}$, and $q W \subset U_{0}$. We denote (again) $U=q W$ and $V=\bar{f} W$. Then $g=\left.f\right|_{U}: U \rightarrow V$ is a proper branched cover with multiplicity 3 and we have the diagram

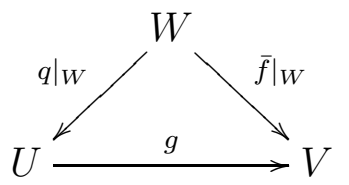

where $q \mid W$ and $\bar{f} \mid W$ are normal branched coverings.

From the monodromy triangle of $g$, we obtain the diagram

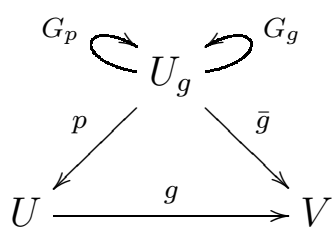

where $\bar{g}$ is the normalization of $g, G_{g}$ is the monodromy group of $g$, and $G_{p} \subset G_{g}$ is the monodromy group of $p$.

Further, by minimality of the monodromy factorization [1, Section 2.2], there exists a branched covering $r: W \rightarrow U_{g}$ and a commutative diagram

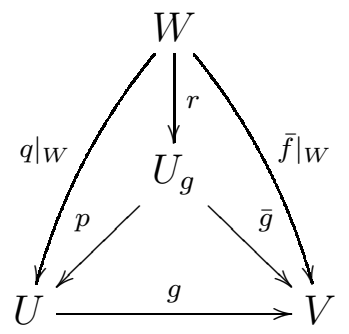

Since $g$ has multiplicity 3 , the monodromy group $G_{g}$ is isomorphic to a subgroup of the symmetric group $S_{3}$. Since $G_{g}$ acts transitively on $\bar{g}^{-1}\left(y_{0}\right)$, we have $\left|G_{g}\right| \geq 3$. Thus either $\left|G_{g}\right|=3$ or $\left|G_{g}\right|=6$, i.e. $G_{g} \cong \mathbf{Z}_{3}$ or $G_{g} \cong S_{3}$.

Suppose $G_{g} \cong \mathbf{Z}_{3}$. Then the local monodromy of $\bar{g}$ at $f(x)$ is abelian and, by Theorem $6, \bar{g} B_{\bar{g}}$ has dimension $n-2$ at $f(x)$. Since $\bar{g} B_{\bar{g}} \subset f B_{f}$, we have that $f B_{f}$ has local dimension at $f(x)$ at least $n-2$.

Suppose now that $G_{g} \cong S_{3}$. We show that $x \in p B_{p}$ and that the local monodromy of $p$ at $x$ is abelian. Then, by Theorem $6, p B_{p}$ has local dimension at least $n-2$ at $x$.

Since $\bar{g}$ has multiplicity 6 and $g$ has multiplicity 3, the branched cover $p$ has multiplicity 2. Thus $G_{p} \cong \mathbf{Z}_{2}$. Moreover, $\mathcal{G}_{p}(x) \cong \mathbf{Z}_{2}$ and, in particular, the local 
monodromy of $p$ at $x \in p B_{p}$ is abelian. Indeed, since $q^{-1}(x) \cap W=\{z\}$, we have that $p^{-1}(x)=\{r(z)\}$. Thus $G_{p}$ fixes $r(z)$.

Since $p B_{p}$ has has local dimension at least $n-2$ at $x$, we have, by the ChurchHemmingsen theorem [7, Corollary 2.3], that $f\left(p B_{p}\right)$ has local dimension at least $n-2$ at $f(x)$. Since $f\left(p B_{p}\right) \subset f B_{f}$, the proof is complete.

\section{Application to toroidal Cantor sets}

We finish an application of Theorem 6 to branched covers branching over toroidal Cantors sets. A Cantor set $C$ in a 3-manifold $M$ is toroidal if every (finite) covering $\left\{U_{i}\right\}_{i \geq 1}$ of $C$ has a (finite) refinement $\left\{T_{j}\right\}_{j \geq 1}$ consisting of mutually disjoint domains $T_{j}$ for which $\partial T_{j}$ is a 2-torus, i.e. $\partial T_{j} \approx S^{1} \times S^{1}$, and $C \cap \partial T_{j}=\emptyset$ for each $j \geq 1$.

We begin with a proposition; in what follows, we define the boundary $\partial X$ of a Fox-completion $X$ of a manifold to be the boundary $\partial X^{o}$ of the regular part $X^{o}$ of $X$.

Proposition 9. Let $X$ be a compact Fox-completion of a 3-manifold having connected boundary $\partial X, T$ a compact 3-manifold with boundary $\partial T \approx S^{1} \times S^{1}$, and $f: X \rightarrow T$ a normal branched covering. If $\left.f\right|_{\partial X}: \partial X \rightarrow \partial T$ is a covering, then $\operatorname{dim} f B_{f}=1$.

Proof. Clearly the restriction $\left.f\right|_{\partial X}: \partial X \rightarrow \partial T$ is a normal covering. The homomorphism $\operatorname{Deck}(f) \mapsto \operatorname{Deck}\left(\left.f\right|_{\partial X}\right),\left.g \mapsto g\right|_{\partial X}$, is well-defined and injective. Indeed, since $f$ is open, $f^{-1}(\partial T)=\partial X$ and $\left.g\right|_{\partial X}: \partial X \rightarrow \partial X$ is well-defined and belongs to the deck group of $\left.f\right|_{\partial X}$.

To show that the restriction $\left.g \mapsto g\right|_{\partial X}$ is injective, let $g$ and $h$ be deck transformations of $f$ satisfying $\left.g\right|_{\partial X}=\left.h\right|_{\partial X}$. By uniqueness of deck transformations of covering maps, we obtain $\left.g\right|_{X \backslash f^{-1} f B_{f}}=\left.h\right|_{X \backslash f^{-1} f B_{f}}$. Thus $g=h$ by the density of $X \backslash f^{-1} f B_{f}$ in $X$.

Since $\partial X$ is connected and $\left.f\right|_{\partial X}: \partial X \rightarrow \partial T$ is a covering, we conclude that $\partial X$ is a 2-torus and $\left.f\right|_{\partial X}$ is a normal covering. In particular, Deck $\left(\left.f\right|_{\partial X}\right)$ is abelian. Thus $\operatorname{Deck}(f)$ is abelian and the claim follows from Theorem 6 .

As a consequence we obtain that branched covers do not branch over toroidal Cantor sets. We formulate this as follows. Theorem 4 for Antoine's necklaces is a particular case of this corollary.

Corollary 10. Let $M$ and $N$ be 3-manifolds and $f: M \rightarrow N$ a locally normal branched cover so that $f B_{f}$ is contained in a toroidal Cantor set. Then $f$ is a covering map.

Proof. Let $y \in f B_{f}, x \in f^{-1}(y)$, and $T \subset N$ a neighborhood of $y$ so that $\partial T$ is a 2-torus in $N \backslash f B_{f}, f^{-1}(N \backslash T)$ is connected, the $x$-component $H_{x}$ of $f^{-1} T$ is contained in an interior of an $n$-cell $C$ in $M$ and $\left.f\right|_{H_{x}}: H_{x} \rightarrow T$ is a normal branched covering. Note that, e.g. by the Alexander duality, each boundary component of $\partial H_{x}$ separates $C$ into exactly two connected components. Since $M \backslash H_{x} \supset f^{-1}(N \backslash T)$ is connected, we conclude that the boundary $\partial H_{x}$ is connected. On the other hand, since $\left.f\right|_{\partial H_{x}}: \partial H_{x} \rightarrow \partial T$ is a covering, we have that $\partial H_{x}$ is a 2-torus. Thus the mapping $\left.f\right|_{H_{x}}: H_{x} \rightarrow T$ satisfies the conditions of Proposition 9. 


\section{References}

[1] Aaltonen, M.: Monodromy representations of completed coverings. - Rev. Mat. Iberoam. $32: 2,2016,533-570$.

[2] Berstein, I., and A. L. Edmonds: The degree and branch set of a branced covering. - Invent. Math. 45:3, 1978, 213-220.

[3] Borel, A.: Seminar on transformation groups. - Ann. of Math. Stud. 46, Princeton Univ. Press, Princeton, N.J., 1960.

[4] Cannon, J. W.: The recognition problem: what is a topological manifold? - Bull. Amer. Math. Soc. 84:5, 1978, 832-866.

[5] Černavskil̆, A. V.: Finite-to-one open mappings of manifolds. - Mat. Sb. (N.S.) 65 (107), 1964, 357-369 (in Russian).

[6] Church, P. T.: Discrete maps on manifolds. - Michigan Math. J. 25:3, 1978, 351-357.

[7] Church, P. T., and E. Hemmingsen: Light open maps on $n$-manifolds. - Duke Math. J. 27, 1960, 527-536.

[8] Edmonds, A. L.: Branched coverings and orbit maps. - Michigan Math. J. 23:4, 1976, $289-301$.

[9] EdwARDs, R. D.: Suspensions of homology spheres. - arXiv: math/0610573, 2006.

[10] Engelking, R.: Dimension theory. - North-Holland Publishing Co., Amsterdam-Oxford-New York; PWN-Polish Scientific Publishers, Warsaw, 1978.

[11] Fox, R. H.: Covering spaces with singularities. - In: A symposium in honor of S. Lefschetz, Princeton Univ. Press, Princeton, N.J., 1957, 243-257.

[12] Heinonen, J., and S. Rickman: Quasiregular maps $\mathbf{S}^{3} \rightarrow \mathbf{S}^{3}$ with wild branch sets. - Topology $37: 1,1998,1-24$.

[13] Heinonen, J., and S. Rickman: Geometric branched covers between generalized manifolds. - Duke Math. J. 113:3, 2002, 465-529.

[14] Massey, W. S.: A basic course in algebraic topology. - Grad. Texts in Math. 127, SpringerVerlag, New York, 1991.

[15] Montesinos-Amilibia, J. M.: Branched coverings after Fox. - Bol. Soc. Mat. Mexicana (3) $11: 1,2005,19-64$.

[16] Montesinos Amilibia, J. M.: On some open 3-manifolds that are branched coverings of the Poincaré homology sphere. - Bol. Soc. Mat. Mexicana (3) 20:2, 2014, 505-521.

[17] Pankka, P., K. Rajala, and J.-M. Wu: Quasiregular ellipticity of open and generalized manifolds. - Comput. Methods Funct. Theory 14:2-3, 2014, 383-398.

[18] VÄIsÄLÄ, J.: Discrete open mappings on manifolds. - Ann. Acad. Sci. Fenn. Ser. A I 392, 1966, $1-10$.

[19] Whyburn, G. T.: Analytic topology. - Amer. Math. Soc. Colloq. Publ. XXVIII, Amer. Math. Soc., Providence, R.I., 1963.

Received 16 May $2016 \bullet$ Accepted 14 October 2016 